\title{
G-quadruplexes and DNA damage in colorectal cancer tumorigenesis
}

Victoria Sanchez-Martin ${ }^{1,2,3}$; Michael Eibl1 ${ }^{1}$; Sonia Morales-Escribano ${ }^{1}$; Ángel Linde-Rodríguez 1,2; Virginia Pérez-Carrasco ${ }^{1,2}$; Matilde Ortiz-González ${ }^{1,4}$; Ana Soriano-Lerma ${ }^{1}$; Miguel Soriano-Rodríguez ${ }^{1,4}$; José Antonio García-Salcedo ${ }^{1,2}$

GENYO. Centre for Genomics and Oncological Research: Pfizer/University of Granada/Andalusian Regional Government, Granada, 18016, Spain

Microbiology Unit, Biosanitary Research Institute IBS-Granada, University Hospital Virgen de las Nieves, Granada, 18014, Spain.

${ }^{3}$ Department of Biochemistry, Molecular Biology III and Immunology, University of Granada, Granada, 18016, Spain.

Center for Intensive Mediterranean Agrosystems and Agri-food Biotechnology (CIAMBITAL), University of Almeria, Almeria, 04001, Spain.

\section{INTRODUCTION}

\section{OBJECTIVES}

- Non-canonical four-stranded G-quadruplex (G4) structures form through self-recognition of guanines into stacked tetrads within chromatin DNA.

- Considerable evidences exist for G4s formation in vitro and have linked G4 formation with key biological processes ranging from transcription and translation to genome instability and cancer. - G4s have not been exploited for colorectal cancer (CRC) treatment.

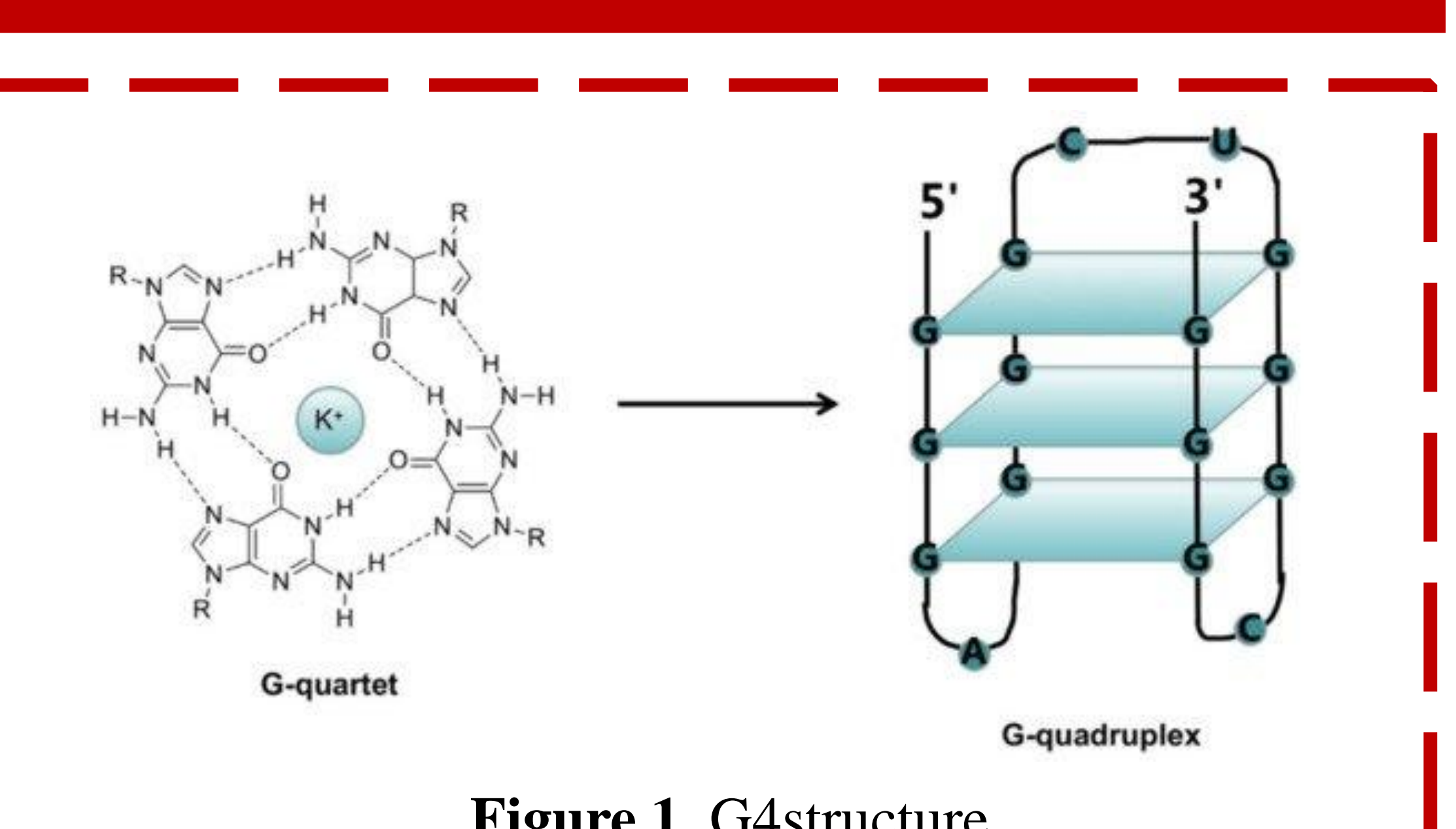

WORKFLOW
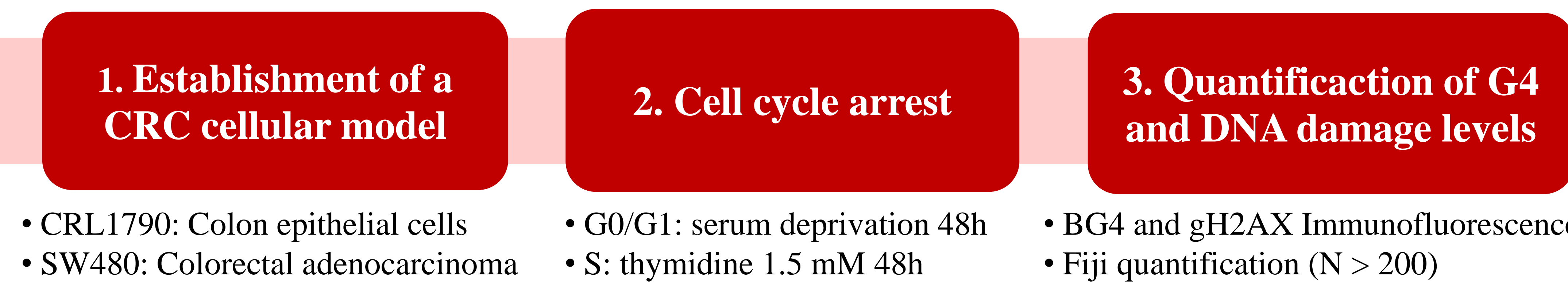

Figure 1. G4structure.

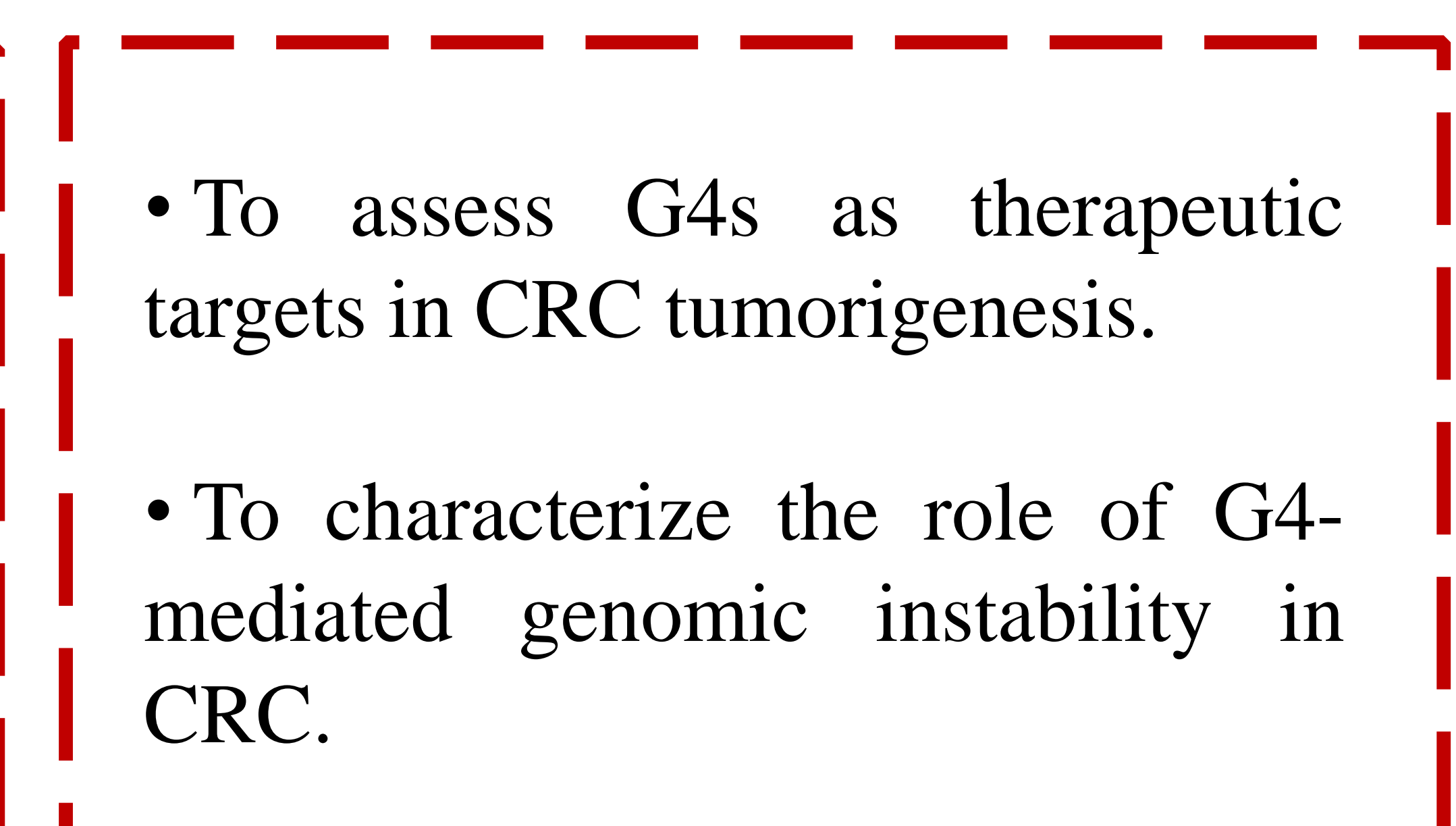

\section{RESULTS}

Figure 2. G4s abundance increases in CRC. Images showing BG4 foci (green) in CRL1790 and SW480 cell nuclei (blue) to detect G4s abundance at G0/G1 and S phases. Respective Fiji quantification is shown in the right pannel.
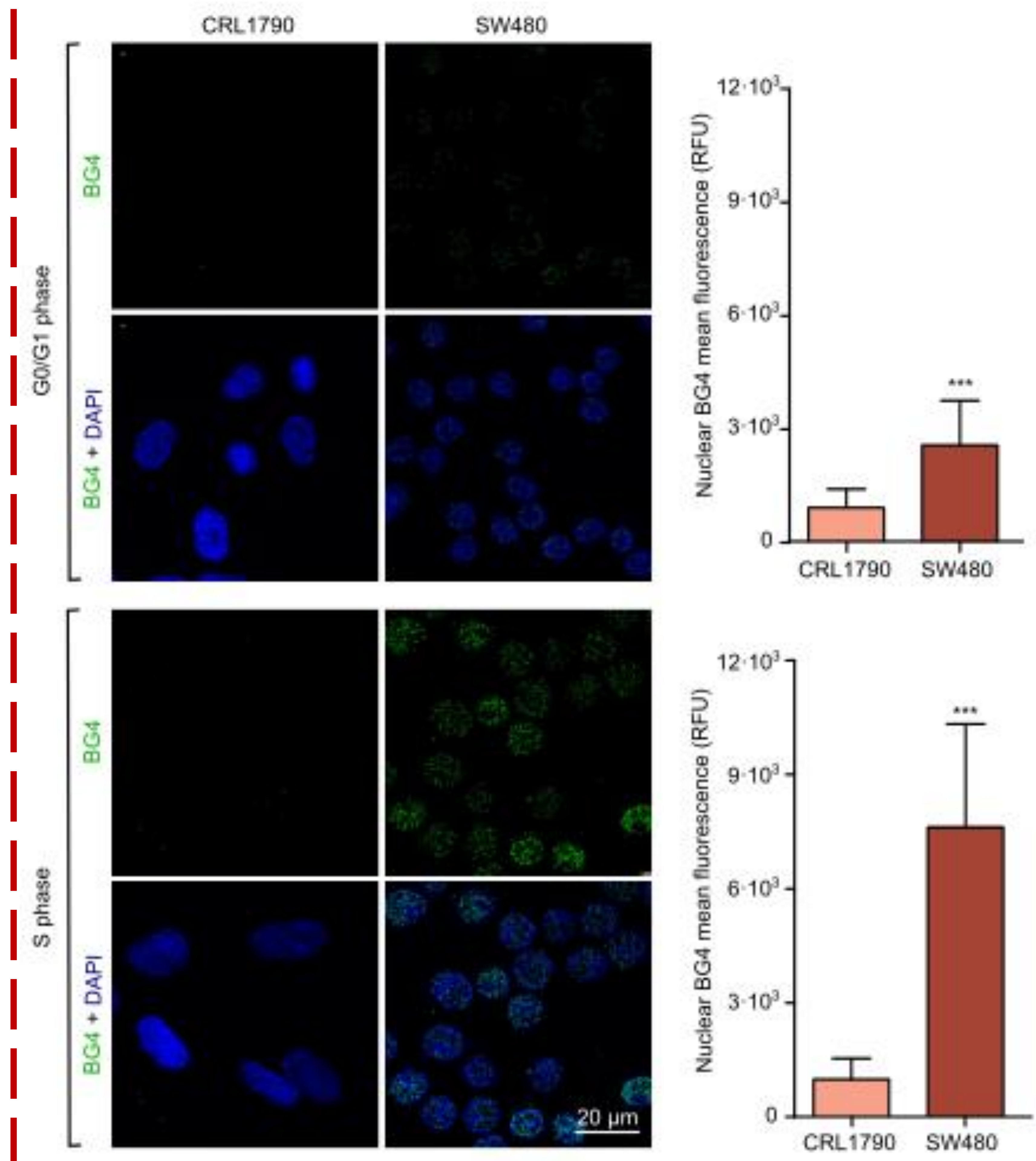

Figure 5. IC50 values for G4ligands in CRC. IC50 values represent G4-ligand concentration inhibiting cell growth by $50 \%$ and are expressed as mean \pm standard deviation.

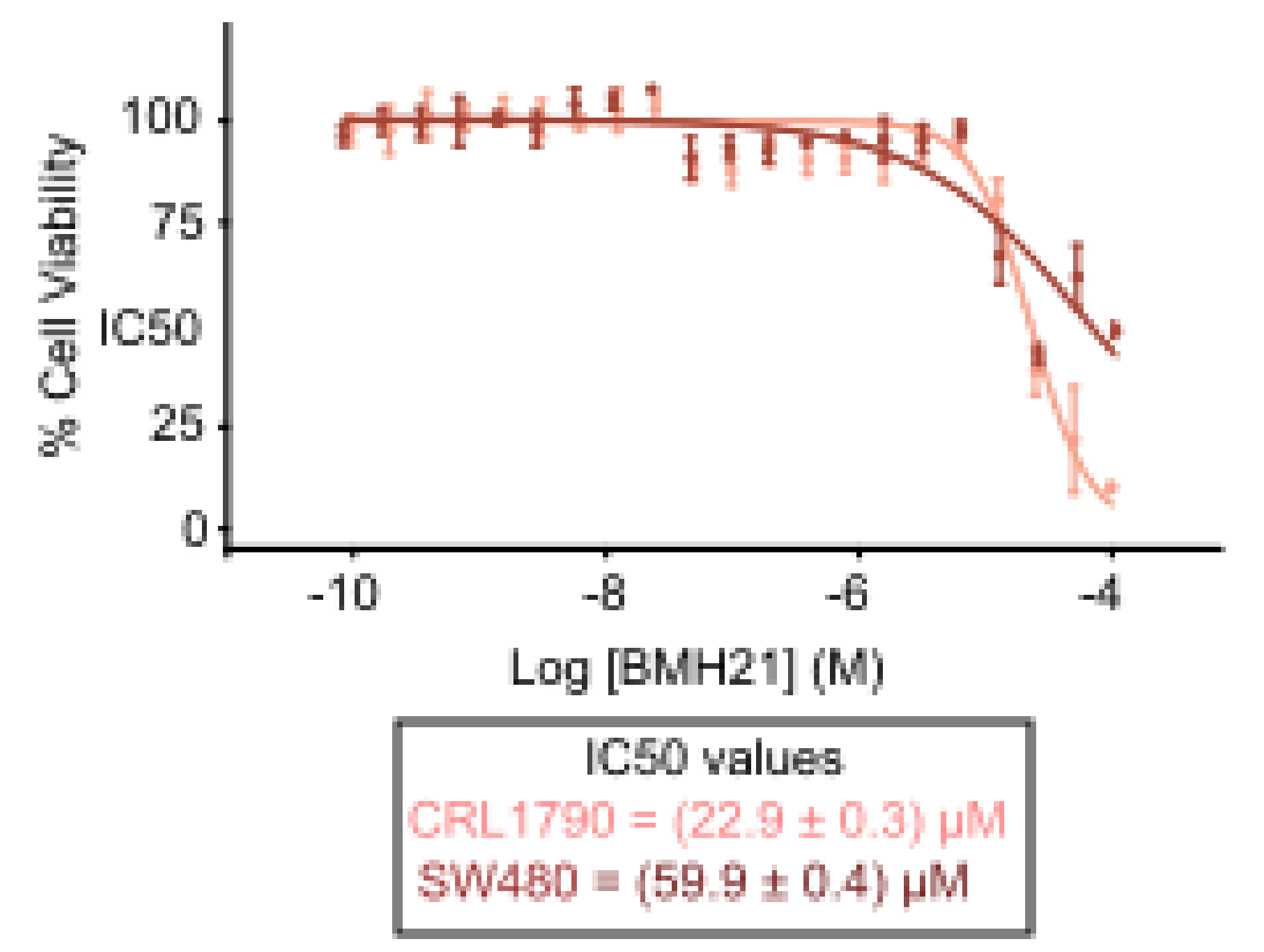$$
\text { deviaton. }
$$

4. Investigation of relationship between $\mathrm{G} 4 \mathrm{~s}$ and DNA damage

- BG4 and gH2AX colocalization analysis
5. Evaluation of G4s as therapeutical targets

- Resazurin method at $48 \mathrm{~h}$ upon treatment with G4s-ligands
Figure 3. DNA damage increases in CRC. Images showing gH2AX foci (red) in CRL1790 and SW480 cell nuclei (blue) to detect gH2AX abundance at G0/G1 and $\mathrm{S}$ phases. Respective Fiji quantification is shown in the right pannel.
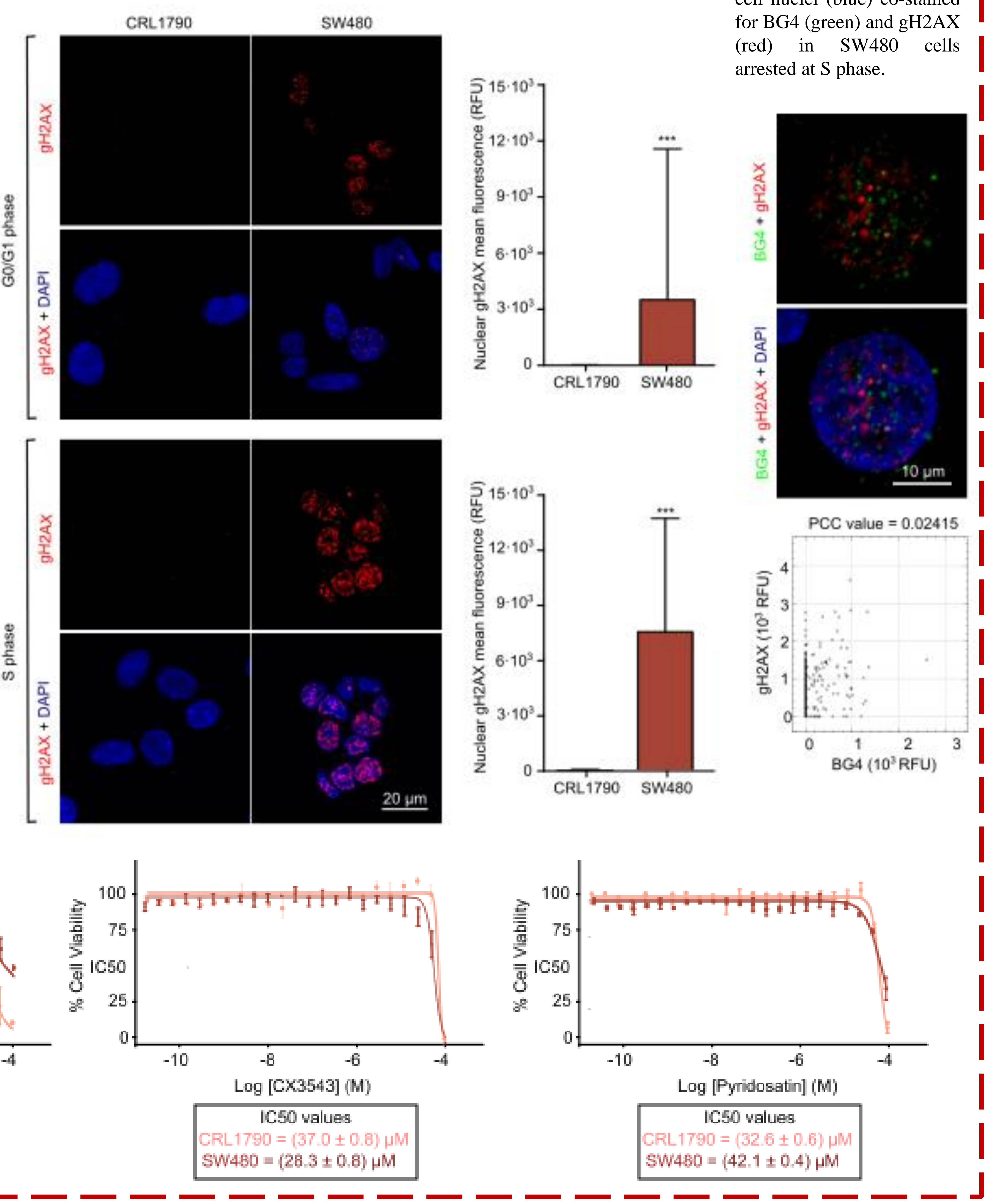
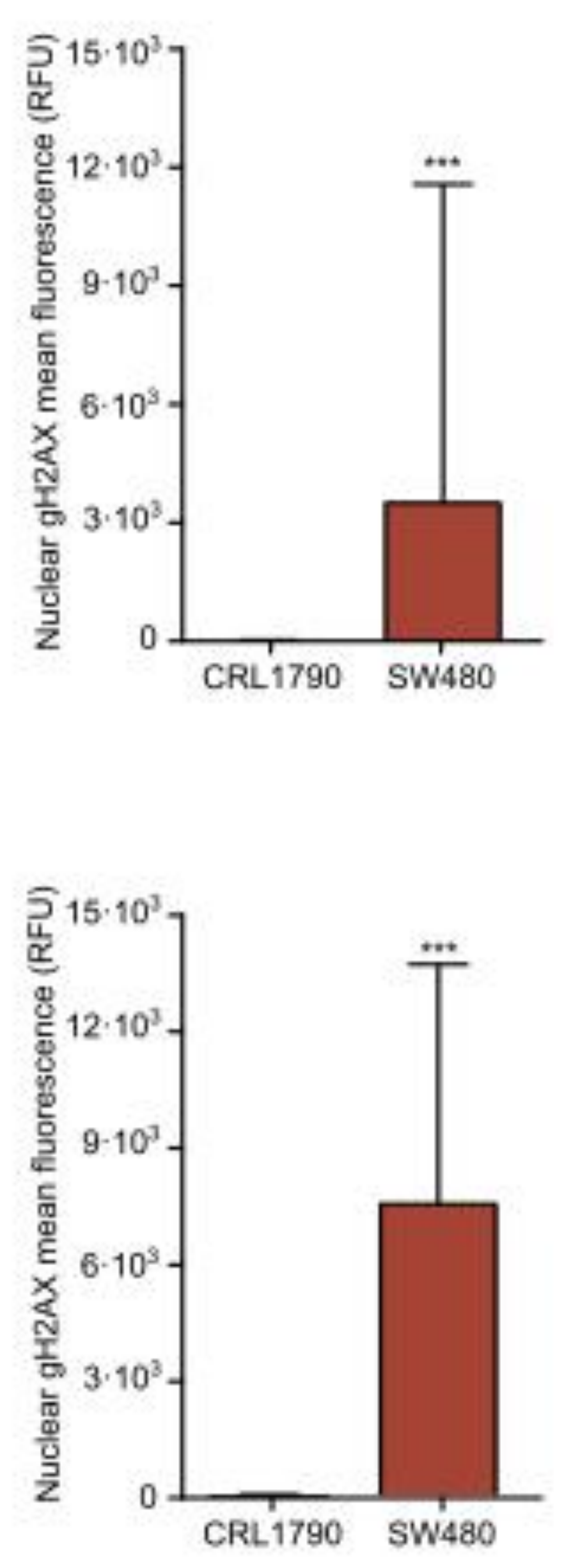

PCC value $=0.02415$

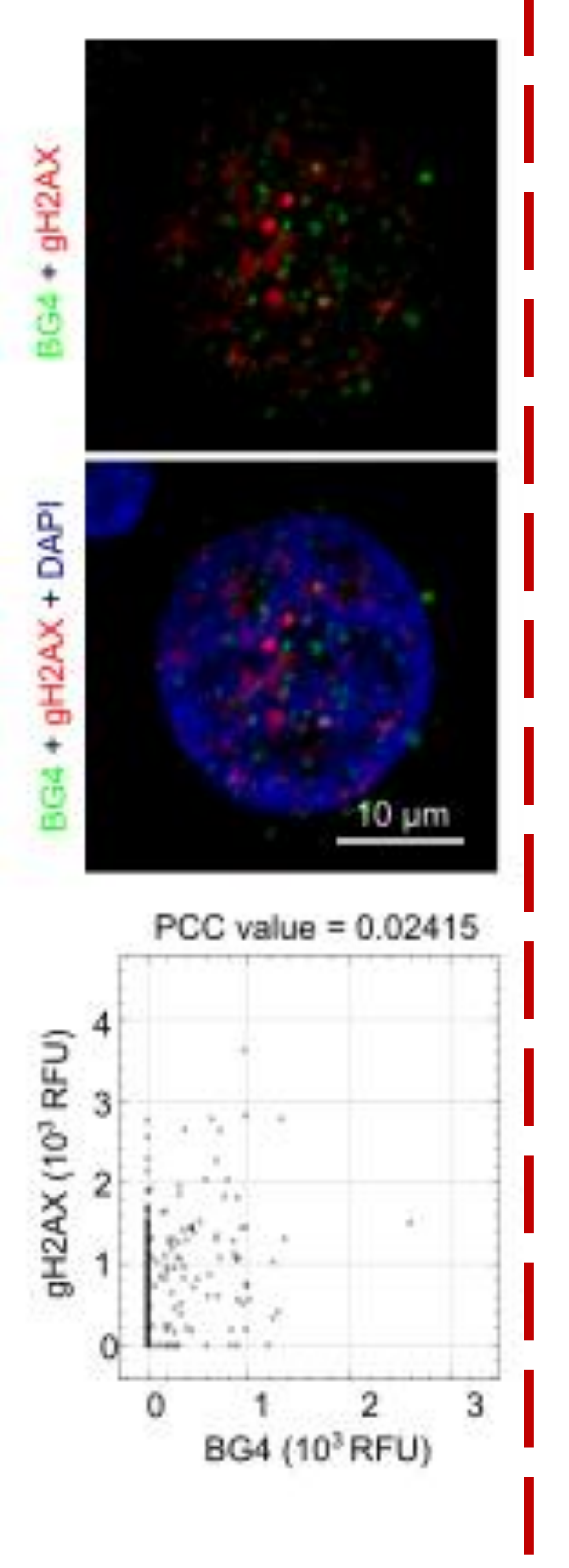

Figure 4. DNA damage is only partially linked to G4s presence. Images of cell nuclei (blue) co-stained for BG4 (green) and $\mathrm{gH} 2 \mathrm{AX}$ (red) in SW480 cells arrested at $\mathrm{S}$ phase.

\section{CONCLUSIONS}

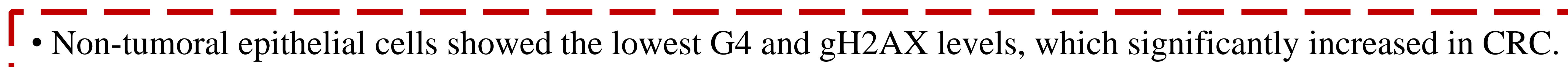

- Genome instability in CRC was only partially explained by G4s presence. 